\title{
Laplace ultradistributions on a half line and a strong quasi-analyticity principle
}

\author{
by GRzEGorz ŁysiK (Warszawa)
}

\begin{abstract}
Several representations of the space of Laplace ultradistributions supported by a half line are given. A strong version of the quasi-analyticity principle of Phragmén-Lindelöf type is derived.

The theory of ultradistributions was founded by Buerling and Roumieu in the sixties as a generalization of the theory of Schwartz distributions. Since then it was extensively studied by many authors: Björk, Braun, Komatsu, Meise, Pilipović, Taylor , ..., to mention but a few. The most systematic treatment was presented by Komatsu [2], [3]. He derived, in particular, the boundary value representation of the space $D^{\left(M_{p}\right) \prime}(\Omega)$ of ultradistributions on an open set $\Omega \subset \mathbb{R}^{n}$, structure theorems for $D^{\left(M_{p}\right)^{\prime}}(\Omega)$ and described the image of the space $D_{K}^{\left(M_{p}\right) \prime}$ of ultradistributions with compact support in $K$ under the Fourier-Laplace transformation. Following his approach Pilipović [9] recently introduced and investigated the space $S^{\left(M_{p}\right)^{\prime}}(\mathbb{R})$ of tempered ultradistributions. On the other hand, in the study of the Laplace transformation it is convenient to consider the space $L_{(\omega)}^{\prime}(\Gamma)$ of Laplace distributions of type $\omega \in \mathbb{R}$ supported by a half line $\Gamma$. Since in the logarithmic variables the Laplace transformation is the Mellin transformation we refer here to the book of Szmydt and Ziemian [11], where the latter transformation was systematically studied following the approach of Zemanian [12].

The aim of the present paper is to unify the theory of ultradistributions with that of Laplace distributions. We present it in the case of the space $L_{(\omega)}^{\left(M_{p}\right) \prime}(\Gamma)$ of Laplace ultradistributions of Buerling type. Our theory is based on the Seeley type extension theorems for ultradifferentiable functions re-
\end{abstract}

1991 Mathematics Subject Classification: Primary 46F12, 44A10, 30D15.

Key words and phrases: ultradistributions, boundary values, quasi-analyticity. Supported by KBN grant No. 2104591091. 
cently proved by Langenbruch [4] and Meise and Taylor [7]. We describe the image of the space $L_{(\omega)}^{\left(M_{p}\right) \prime}(\Gamma)$ under the Laplace, Taylor and (modified) Cauchy transformations. In the latter case we follow the method of Morimoto [8]. As an application of our theory we give, in the final section, a version of the quasi-analyticity principle of Phragmén-Lindelöf type. It says that a function holomorphic and of exponential type in the half plane $\{\operatorname{Re} z>0\}$ vanishes if it satisfies some growth conditions along vertical lines and decreases superexponentially along a ray in $\{\operatorname{Re} z>0\}$.

0. Notation. Let $t>0$. We denote by $\widetilde{B}(t)$ the universal covering of the punctured disc $B(t) \backslash\{0\}$ and by $\widetilde{\mathbb{C}}$ that of $\mathbb{C} \backslash\{0\}$. We treat $\widetilde{B}(t)$ and $\widetilde{\mathbb{C}}$ as Riemann manifolds. Recall that any point $x \in \widetilde{B}(t)$ can be written in the form $x=|x| \exp i \arg x$ with $|x|<t$.

We denote by $\mu: \mathbb{C} \rightarrow \widetilde{\mathbb{C}}$ the biholomorphism

$$
\mu(z)=e^{-z} \quad \text { for } z \in \mathbb{C},
$$

i.e. $\mu(z)=x \in \widetilde{\mathbb{C}}$ with $|x|=e^{-\operatorname{Re} z}, \arg x=-\operatorname{Im} z$. Then the inverse mapping $\mu^{-1}: \widetilde{\mathbb{C}} \rightarrow \mathbb{C}$ is given by

$$
\mu^{-1}(x)=-\ln x \quad \text { for } x \in \widetilde{\mathbb{C}} .
$$

Let $v \in \mathbb{R}$. We set

$$
\Gamma_{v}=[v, \infty) \quad \text { and } \quad I_{v}=\left(0, e^{-v}\right] .
$$

Observe that $I_{v}=\mu\left(\Gamma_{v}\right)$. In the following we omit the subscript $v$ as long as it is fixed. For $z \in \mathbb{C}$ we define the function $\exp _{z}: \mathbb{R} \rightarrow \mathbb{C}$ by

$$
\exp _{z} y=e^{y z}, \quad y \in \mathbb{R} .
$$

For $A \subset \mathbb{C}$ we set

$$
A_{\varepsilon}=\{z \in \mathbb{C}: \operatorname{dist}(z, A)<\varepsilon\}, \quad \varepsilon>0 .
$$

We write $D$ for the differential operator $d / d x$.

Let $\left\{P_{\tau}\right\}_{\tau \in T}$ be a family of multivalued vector spaces. Then $\lim _{\tau \in T} T_{\tau}$ (resp. $\lim _{\tau \in T} P_{\tau}$ ) denotes the inductive limit (resp. projective limit) of $P_{\tau}$, $\tau \in T$.

$\mathcal{O}(W)$ denotes the set of holomorphic functions on an open subset $W$ of some Riemann manifold. The value of a functional $S$ on a test function $\varphi$ is denoted by $S[\varphi]$.

1. Laplace ultradistributions on a half line. Let $\left(M_{p}\right)_{p \in \mathbb{N}_{0}}$ be a sequence of positive numbers. Throughout the paper we assume that $\left(M_{p}\right)$ satisfies the following conditions: 
(M.1) (Logarithmic convexity)

$$
M_{p}^{2} \leq M_{p-1} M_{p+1} \quad \text { for } p \in \mathbb{N}
$$

(M.2) (Stability under ultradifferential operators) There are constants $A, H$ such that

$$
M_{p} \leq A H^{p} \min _{0 \leq q \leq p} M_{q} M_{p-q} \quad \text { for } p \in \mathbb{N}_{0} ;
$$

(M.3) (Strong non-quasi-analyticity) There is a constant $A$ such that

$$
\sum_{q=p+1}^{\infty} \frac{M_{q-1}}{M_{q}} \leq A p \frac{M_{p}}{M_{p+1}} \quad \text { for } p \in \mathbb{N} .
$$

Some results remain valid when (M.2), (M.3) are replaced by the following weaker conditions:

(M.2') (Stability under differential operators) There are constants $A, H$ such that

$$
M_{p+1} \leq A H^{p} M_{p} \quad \text { for } p \in \mathbb{N}_{0} ;
$$

$($ M.3' $\quad$ (Non-quasi-analyticity)

$$
\sum_{p=1}^{\infty} \frac{M_{p-1}}{M_{p}}<\infty .
$$

Define

$$
m_{p}=M_{p} / M_{p-1} \quad \text { for } p \in \mathbb{N} .
$$

Then (M.1) is equivalent to saying that the sequence $m_{p}$ is non-decreasing, and by (M.3') it follows that $m_{p} \rightarrow \infty$.

Note that the condition (M.3') implies the following: for every $h>0$ there exists $\delta>0$ such that

$$
M_{p} h^{p} \geq \delta \quad \text { for } p \in \mathbb{N}_{0},
$$

which is equivalent to the finiteness of the associated function $M$ defined by

$$
M(\varrho)=\sup _{p \in \mathbb{N}_{0}} \ln \frac{\varrho^{p} M_{0}}{M_{p}} \quad \text { for } \varrho>0 .
$$

If $M_{p} / p$ ! satisfies (1) the growth function $M^{*}$ is defined by

$$
M^{*}(\varrho)=\sup _{p \in \mathbb{N}_{0}} \ln \frac{\varrho^{p} p ! M_{0}}{M_{p}} \quad \text { for } \varrho>0 .
$$

Example 1. The Gevrey sequence of order $s>1$ is defined by $M_{p}=$ $(p !)^{s}, p \in \mathbb{N}_{0}$. It satisfies all conditions (M.1)-(M.3) and $M(\varrho) \sim \varrho^{1 / s}$, $M^{*}(\varrho) \sim \varrho^{1 / s-1}$ as $\varrho \rightarrow \infty$. 
Remark 1. It follows from Lemma 4.1 of [2] that if $M_{p}$ satisfies (M.1) and $\left(\mathrm{M} .3^{\prime}\right)$ then the associated function $M$ is sublinear, i.e. $M(\varrho) / \varrho \rightarrow 0$ as $\varrho \rightarrow \infty$.

Remark 2. If $M_{p}$ satisfies (M.1) and (M.3') then

$$
\lim _{p \rightarrow \infty}\left(M_{p} / p !\right)^{1 / p}=\infty .
$$

Proof. Take any $l<\infty$. Then by (M.1) and (M.3') there exists $p_{l} \in \mathbb{N}$ such that $M_{p} \geq l p M_{p-1}$ for $p \geq p_{l}$. Hence

$$
M_{p} \geq C_{l} \cdot l^{p} \cdot p ! \quad \text { for } p \geq p_{l}, \quad \text { where } C_{l}=\frac{M_{p_{l}-1}}{M_{0}\left(p_{l}-1\right) !} l^{1-p_{l}},
$$

and we easily get (4).

Definition. Let $\Gamma=[v, \infty)$ with $v \in \mathbb{R}$. The space $D^{\left(M_{p}\right)^{\prime}}(\Gamma)$ of ultradistributions on $\Gamma$ of class $\left(M_{p}\right)$ is defined as the dual space of

$$
D^{\left(M_{p}\right)}(\Gamma)=\lim _{K \subset \Gamma} \lim _{h>0} D_{K, h}^{\left(M_{p}\right)}(\Gamma)
$$

where for any compact set $K \subset \Gamma$ and $h>0$,

$$
\begin{aligned}
& D_{K, h}^{\left(M_{p}\right)}(\Gamma) \\
& \quad=\left\{\varphi \in C^{\infty}(\Gamma): \operatorname{supp} \varphi \subset K \text { and }\|\varphi\|_{K, h}^{\left(M_{p}\right)}=\sup _{y \in K} \sup _{\alpha \in \mathbb{N}_{0}} \frac{\left|D^{\alpha} \varphi(y)\right|}{h^{\alpha} M_{\alpha}}<\infty\right\} .
\end{aligned}
$$

By $\varphi \in C^{\infty}(\Gamma)$ we mean a restriction to $\Gamma$ of some function $\widetilde{\varphi} \in C^{\infty}(\mathbb{R})$.

Definition. Let $\omega \in \mathbb{R} \cup\{\infty\}$. We define the space $L_{(\omega)}^{\left(M_{p}\right) \prime}(\Gamma)$ of Laplace ultradistributions as the dual space of

$$
L_{(\omega)}^{\left(M_{p}\right)}(\Gamma)=\varliminf_{a<\omega} L_{a}^{\left(M_{p}\right)}(\Gamma)
$$

where for any $a \in \mathbb{R}$,

$$
\begin{aligned}
& L_{a}^{\left(M_{p}\right)}(\Gamma)=\lim _{h>0} L_{a, h}^{\left(M_{p}\right)}(\Gamma), \\
& L_{a, h}^{\left(M_{p}\right)}(\Gamma)=\left\{\varphi \in C^{\infty}(\Gamma):\|\varphi\|_{a, h}^{\left(M_{p}\right)}=\sup _{y \in \Gamma} \sup _{\alpha \in \mathbb{N}_{0}} \frac{\left|e^{-a y} D^{\alpha} \varphi(y)\right|}{h^{\alpha} M_{\alpha}}<\infty\right\} .
\end{aligned}
$$

Lemma 1. Assume that $\left(M_{p}\right)$ satisfies (M.1) and (M.3'). Then $D^{\left(M_{p}\right)}(\Gamma)$ is a dense subspace of $L_{(\omega)}^{\left(M_{p}\right)}(\Gamma)$. Thus, $L_{(\omega)}^{\left(M_{p}\right) \prime}(\Gamma)$ is a subspace of the space of ultradistributions $D^{\left(M_{p}\right) \prime}(\Gamma)$.

Proof. Making a translation if necessary we can assume that $\Gamma=\overline{\mathbb{R}}_{+}$. Let $\varphi \in L_{(\omega)}^{\left(M_{p}\right)}(\Gamma)$. Then there exist $a<b<\omega$ such that $\varphi \in L_{a}^{\left(M_{p}\right)}(\Gamma) \subset$ $L_{b}^{\left(M_{p}\right)}(\Gamma)$. By the Denjoy-Carleman-Mandelbrojt theorem (cf. [2], [6]) there 
exists a function $\psi \in D^{\left(M_{p}\right)}(\Gamma)$ such that $0 \leq \psi(y) \leq 1$ for $y \in \Gamma, \psi(y)=1$ for $0 \leq y \leq 1$ and $\psi(y)=0$ for $y \geq 2$. Put $\varphi_{\nu}(y)=\psi(y / \nu) \varphi(y)$ for $y \in \Gamma, \nu \in \mathbb{N}$. Then $\varphi_{\nu} \in D^{\left(M_{p}\right)}(\Gamma)$ and we shall show that $\varphi_{\nu} \rightarrow \varphi$ in $L_{b}^{\left(M_{p}\right)}(\Gamma)$ as $\nu \rightarrow \infty$. To this end take any $h>0$. Noting that (M.1) implies $M_{q} M_{p-q} \leq M_{0} M_{p}$ for $0 \leq q \leq p$, by the Leibniz formula we get

$$
\begin{aligned}
\| \varphi_{\nu} & -\varphi \|_{b, h}^{\left(M_{p}\right)}=\sup _{y \in \Gamma} \sup _{\alpha \in \mathbb{N}_{0}} \frac{\left|e^{-b y} D^{\alpha}(\varphi(y)(\psi(y / \nu)-1))\right|}{h^{\alpha} M_{\alpha}} \\
\leq & \sup _{y \in \Gamma} \sup _{\alpha \in \mathbb{N}_{0}} \frac{e^{-a y}\left|D^{\alpha} \varphi(y)\right|}{h^{\alpha} M_{\alpha}} e^{(a-b) y}|\psi(y / \nu)-1| \\
& +\sup _{y \in \Gamma} \sup _{\alpha \in \mathbb{N}} \sum_{0 \leq \beta<\alpha}\left(\begin{array}{l}
\alpha \\
\beta
\end{array}\right) \frac{e^{-a y}\left|D^{\beta} \varphi(y)\right|}{h^{\beta} M_{\beta}} \cdot \frac{e^{(a-b) y}\left|D^{\alpha-\beta}(\psi(y / \nu)-1)\right| M_{0}}{h^{\alpha-\beta} M_{\alpha-\beta}} .
\end{aligned}
$$

Since $\psi(y / \nu)=1$ for $0 \leq y \leq \nu$ the first summand tends to zero as $\nu \rightarrow \infty$. Put $K=[1,2]$. Then for $\beta<\alpha$ and any $h_{1}>0$ we have

$$
\left|D^{\alpha-\beta}(\psi(y / \nu)-1)\right|=\left|\nu^{-(\alpha-\beta)} \psi^{(\alpha-\beta)}(y / \nu)\right| \leq \nu^{-1}\|\psi\|_{K, h_{1}}^{\left(M_{p}\right)} h_{1}^{\alpha-\beta} M_{\alpha-\beta} .
$$

We also have for any $h_{2}>0$ and $\beta \geq 0, e^{-a y}\left|D^{\beta} \varphi(y)\right| \leq\|\varphi\|_{a, h_{2}}^{\left(M_{p}\right)} h_{2}^{\beta} M_{\beta}$. So the second summand is bounded by

$$
\begin{aligned}
& \frac{M_{0} e^{(a-b) \nu}}{\nu} \sup _{\alpha \in \mathbb{N}} \sum_{\beta<\alpha}\left(\begin{array}{c}
\alpha \\
\beta
\end{array}\right)\|\varphi\|_{a, h_{2}}^{\left(M_{p}\right)}\left(\frac{h_{2}}{h}\right)^{\beta}\|\psi\|_{K, h_{1}}^{\left(M_{p}\right)}\left(\frac{h_{1}}{h}\right)^{\alpha-\beta} \\
& \quad \leq \frac{M_{0}}{\nu}\|\varphi\|_{a, h_{2}}^{\left(M_{p}\right)}\|\psi\|_{K, h_{1}}^{\left(M_{p}\right)} \quad \text { if } h_{2}+h_{1} \leq h
\end{aligned}
$$

and thus tends to zero as $\nu \rightarrow \infty$, proving the lemma.

EXAMPLE 2. Let $\left(M_{p}\right)$ satisfy (1). Then the function

$$
\Gamma \ni y \rightarrow \exp _{z} y=e^{y z}
$$

belongs to $L_{(\omega)}^{\left(M_{p}\right)}(\Gamma)$ if and only if $\operatorname{Re} z<\omega$. Furthermore, in this case for any $a<\omega$ and $h>0$ we have

$$
\left\|\exp _{z}\right\|_{a, h}^{\left(M_{p}\right)}=M_{0}^{-1} \exp \{(\operatorname{Re} z-a) v+M(|z| / h)\} .
$$

Let $\left(M_{p}\right)$ satisfy (M.1) and (1), and let $z \in \mathbb{C}$. Then the operation of multiplication

$$
\exp _{z}: L_{(\omega)}^{\left(M_{p}\right)}(\Gamma) \rightarrow L_{(\omega+\operatorname{Re} z)}^{\left(M_{p}\right)}(\Gamma)
$$

is continuous. Thus the formula

$$
\exp _{z} S[\varphi]=S\left[\exp _{z} \varphi\right] \quad \text { for } \varphi \in L_{(\omega+\operatorname{Re} z)}^{\left(M_{p}\right)}(\Gamma), S \in L_{(\omega)}^{\left(M_{p}\right) \prime}(\Gamma)
$$

defines a continuous operation

$$
\exp _{z}: L_{(\omega)}^{\left(M_{p}\right) \prime}(\Gamma) \rightarrow L_{(\omega-\operatorname{Re} z)}^{\left(M_{p}\right) \prime}(\Gamma) .
$$


Let $\left(M_{p}\right)$ satisfy (M.2). An ultradifferential operator $P(D)$ of class $\left(M_{p}\right)$ is defined by

$$
P(D)=\sum_{\alpha=0}^{\infty} a_{\alpha} D^{\alpha}
$$

where $a_{\alpha} \in \mathbb{C}$ satisfy the following condition: there are constants $K<\infty$ and $C<\infty$ such that

$$
\left|a_{\alpha}\right| \leq C \frac{K^{\alpha}}{M_{\alpha}} \quad \text { for } \alpha \in \mathbb{N}_{0} .
$$

The entire function $\mathbb{C} \ni z \rightarrow P(z)$ is called a symbol of class $\left(M_{p}\right)$. As in the proof of Theorem 2.12 of [2] one can show that an ultradifferential operator of class $\left(M_{p}\right)$ defines linear continuous mappings

$$
P(D): L_{(\omega)}^{\left(M_{p}\right)}(\Gamma) \rightarrow L_{(\omega)}^{\left(M_{p}\right)}(\Gamma), \quad P(D): L_{(\omega)}^{\left(M_{p}\right) \prime}(\Gamma) \rightarrow L_{(\omega)}^{\left(M_{p}\right) \prime}(\Gamma),
$$

where for $S \in L_{(\omega)}^{\left(M_{p}\right) \prime}(\Gamma)$ and $\varphi \in L_{(\omega)}^{\left(M_{p}\right)}(\Gamma)$,

$$
P(D) S[\varphi]=S\left[P^{*}(D) \varphi\right] \quad \text { with } \quad P^{*}(D)=\sum_{\alpha=0}^{\infty}(-1)^{\alpha} a_{\alpha} D^{\alpha} .
$$

For $a \in \mathbb{R}$ and $\omega \in \mathbb{R} \cup\{\infty\}$ we define

$$
Y_{a}=\operatorname{span}\left\{\exp _{c}\right\}_{c \leq a}, \quad Y_{(\omega)}=\bigcup_{a<\omega} Y_{a} .
$$

Proposition 1. Let $b<a$. Then $L_{b}^{\left(M_{p}\right)}(\Gamma)$ is contained in the closure of $Y_{a}$ in $L_{a}^{\left(M_{p}\right)}(\Gamma)$. Thus $Y_{(\omega)}$ is dense in $L_{(\omega)}^{\left(M_{p}\right)}(\Gamma)$.

Proof. Since the multiplication by $\exp _{-a}$ is a continuous isomorphism of $L_{c}^{\left(M_{p}\right)}(\Gamma)$ onto $L_{c-a}^{\left(M_{p}\right)}(\Gamma)$ and $Y_{c}$ onto $Y_{c-a}$, where $c \in \mathbb{R}$, it is sufficient to assume that $a=0$. Let $\varphi \in L_{b}^{\left(M_{p}\right)}(\Gamma)$. It is enough to show that for every $\varepsilon>0$ and $h>0$ there exists $\psi \in Y_{0}$ such that $\|\varphi-\psi\|_{0, h}^{\left(M_{p}\right)}<\varepsilon$. To this end fix $\varepsilon>0$ and $h>0$. By the proof of Lemma 1 there exists $\widetilde{\psi} \in D^{\left(M_{p}\right)}(\Gamma)$ such that $\|\varphi-\widetilde{\psi}\|_{0, h}^{\left(M_{p}\right)}<\varepsilon / 2$. Put $\eta(x)=\psi \circ \mu^{-1}(x)$ for $x \in I$. Then $\eta$ has compact support in $I=\mu(\Gamma)$ and by the Roumieu theorem ([10], Théorème 13), $\eta \in D^{\left(M_{p}\right)}(I)$. By the Weierstrass type theorem ([2], Theorem 7.3) for any $\delta>0$ and $h_{1}>0$ there exists a polynomial $p=\sum_{\nu=0}^{N} c_{\nu} x^{\nu}$ such that

$$
\|\eta-p\|_{\bar{I}, h_{1}}^{\left(M_{p}\right)}<\delta .
$$

Put $\psi(y)=p \circ \mu(y)=\sum_{\nu=0}^{N} c_{\nu} e^{-\nu y}$ for $y \in \Gamma$. Then $\psi \in Y_{0}$ and we shall show that for a suitable choice of $\delta$ and $h_{1},\|\widetilde{\psi}-\psi\|_{0, h}^{\left(M_{p}\right)}<\varepsilon / 2$. To this end 
put $f=\eta-p$. Following the proof of Théorème 13 of [10] one can show that the derivatives $D^{\alpha} f \circ \mu$ are estimated by

$$
\|f\|_{\bar{I}, h_{1}}^{\left(M_{p}\right)} \cdot \sum_{\beta=1}^{\alpha} e^{-v \beta} \frac{M_{\beta}}{\beta !} h_{1}^{\beta} \frac{(\alpha-1) ! \alpha !}{(\alpha-\beta) !(\beta-1) !} .
$$

For $\gamma \in \mathbb{N}_{0}$ put

$$
H_{\gamma}=\sup _{\beta \geq \gamma}\left(\beta ! / M_{\beta}\right)^{1 / \beta} .
$$

Then by Remark 2, $H_{\gamma} \rightarrow 0$ as $\gamma \rightarrow \infty$. Hence we can find $\gamma \in \mathbb{N}_{0}$ and $h_{1}>0$ such that

$$
\left(\sqrt{e^{-v} h_{1}}+\sqrt{H_{\gamma}}\right)^{2} \cdot H<h,
$$

where $H$ is the constant in (M.2). Since by (M.2), $M_{p+q} \leq A H^{p+q} M_{p} M_{q}$ for $p, q \in \mathbb{N}_{0}$ and by (M.1), $M_{\beta} M_{\alpha-\beta} \leq M_{1} M_{\alpha}$ for $0 \leq \beta \leq \alpha$, we get for $\alpha \in \mathbb{N}_{0}, 0 \leq \beta \leq \alpha$,

(11) $M_{\beta} M_{\alpha-\beta+\gamma} \leq M_{\beta} A H^{\alpha-\beta+\gamma} M_{\alpha-\beta} M_{\gamma} \leq C_{\gamma} H^{\alpha} M_{\alpha}$, where $C_{\gamma}=A M_{1} M_{\gamma} \max \left(H^{\gamma}, 1\right)$.

Observe also that

$$
\alpha<\beta(\alpha-\beta+\gamma) \quad \text { for } 1 \leq \beta \leq \alpha, \alpha \in \mathbb{N},
$$

and

$$
\sum_{\beta=1}^{\alpha}\left(\begin{array}{l}
\alpha-1 \\
\beta-1
\end{array}\right)^{2} x^{\beta-1} y^{\alpha-\beta} \leq(\sqrt{x}+\sqrt{y})^{2 \alpha-2} \quad \text { for } \alpha \in \mathbb{N}, x \geq 0, y \geq 0 .
$$

Hence using (8), (9) and (11) we derive for $\alpha \in \mathbb{N}, y \in \Gamma$,

$$
\begin{aligned}
& \left|D^{\alpha} f \circ \mu(y)\right| \\
& \leq\|f\|_{\bar{I}, h_{1}}^{\left(M_{p}\right)} \cdot \sum_{\beta=1}^{\alpha} e^{-v \beta} h_{1}^{\beta} H_{\gamma}^{\alpha-\beta+\gamma} \frac{(\alpha-1) ! \alpha !}{(\alpha-\beta) !(\alpha-\beta+\gamma) ! \beta !(\beta-1) !} M_{\beta} M_{\alpha-\beta+\gamma} \\
& \leq\|f\|_{\bar{I}, h_{1}}^{\left(M_{p}\right)} \cdot C_{\gamma} H_{\gamma}^{\gamma} e^{-v} h_{1} \sum_{\beta=1}^{\alpha}\left(e^{-v} h_{1}\right)^{\beta-1} H_{\gamma}^{\alpha-\beta}\left(\frac{(\alpha-1) !}{(\alpha-\beta) !(\beta-1) !}\right)^{2} H^{\alpha} M_{\alpha} \\
& \leq\|f\|_{\bar{I}, h_{1}}^{\left(M_{p}\right)} \cdot C_{\gamma} H_{\gamma}^{\gamma} e^{-v} h_{1}\left(\sqrt{e^{-v} h_{1}}+\sqrt{H_{\gamma}}\right)^{-2}\left(\left(\sqrt{e^{-v} h_{1}}+\sqrt{H_{\gamma}}\right)^{2} H\right)^{\alpha} M_{\alpha} \\
& \leq \widetilde{C}_{\gamma} M_{\alpha} L^{\alpha}\|f\|_{\bar{I}, h_{1}}^{\left(M_{p}\right)}, \quad \text { where } L=\left(\sqrt{e^{-v} h_{1}}+\sqrt{H_{\gamma}}\right)^{2} H .
\end{aligned}
$$

Finally, choosing $\delta<\varepsilon /\left(2 \widetilde{C}_{\gamma}\right)$ in (7) we get by (10), 


$$
\|\widetilde{\psi}-\psi\|_{0, h}^{\left(M_{p}\right)}=\sup _{y \in \Gamma} \sup _{\alpha \in \mathbb{N}_{0}} \frac{\left|D^{\alpha} f \circ \mu(y)\right|}{h^{\alpha} M_{\alpha}} \leq \sup _{\alpha \in \mathbb{N}_{0}} \frac{\widetilde{C}_{\gamma} L^{\alpha}}{h^{\alpha}}\|f\|_{\bar{I}, h_{1}}^{\left(M_{p}\right)}<\frac{\varepsilon}{2} .
$$

To end this section we quote the following version of

SeEley extension theorem $([4],[7])$. Let $\Gamma=[v, \infty), \Gamma_{1}=\left[v_{1}, \infty\right)$ with $v_{1}<v$ and $a \in \mathbb{R}$. Then there exists a linear continuous extension operator

$$
\mathcal{E}: L_{a}^{\left(M_{p}\right)}(\Gamma) \rightarrow L_{a}^{\left(M_{p}\right)}\left(\Gamma_{1}\right)
$$

such that for every $\varphi \in L_{a}^{\left(M_{p}\right)}(\Gamma)$, $\operatorname{supp} \mathcal{E} \varphi \subset\left(v_{1}, \infty\right)$.

Corollary 1. Let $S$ be a linear functional on $L_{(\omega)}^{\left(M_{p}\right)}(\Gamma)$. Then $S \in$ $L_{(\omega)}^{\left(M_{p}\right) \prime}(\Gamma)$ if and only if for every $a<\omega$ there exist $h>0$ and $C<\infty$ such that

$$
|S[\varphi]| \leq C\|\varphi\|_{a, h}^{\left(M_{p}\right)} \quad \text { for } \varphi \in L_{a}^{\left(M_{p}\right)}(\Gamma)
$$

2. The Paley-Wiener type theorem for Laplace ultradistributions. We assume the conditions (M.1), (M.2) and (M.3). Let $\Gamma=[v, \infty)$ with $v \in \mathbb{R}$. By Example 2 the function $\exp _{z}$ belongs to $L_{(\omega)}^{\left(M_{p}\right)}(\Gamma)$ if and only if $\operatorname{Re} z<\omega$. Hence we can define the Laplace transform of $S \in L_{(\omega)}^{\left(M_{p}\right) \prime}(\Gamma)$ by

$$
\mathcal{L} S(z)=S\left[\exp _{z}\right] \quad \text { for } \operatorname{Re} z<\omega .
$$

Since the mapping

$$
\{\operatorname{Re} z<\omega\} \ni z \rightarrow \exp _{z} \in L_{(\omega)}^{\left(M_{p}\right)}(\Gamma)
$$

is holomorphic, $\mathcal{L} S$ is a holomorphic function on $\{\operatorname{Re} z<\omega\}$.

Define

$$
\begin{aligned}
& \mathcal{O}_{v}^{\left(M_{p}\right)}(\operatorname{Re} z<\omega) \\
& =\{F \in \mathcal{O}(\operatorname{Re} z<\omega): \\
& \quad \text { for every } a<\omega \text { there exist } h>0 \text { and } C<\infty \text { such that } \\
& \quad|F(z)| \leq C \exp \{v \operatorname{Re} z+M(|z| / h)\} \text { for } \operatorname{Re} z \leq a\} .
\end{aligned}
$$

Applying Corollary 1 with $\varphi=\exp _{z}$ and $\operatorname{Re} z \leq a$, by Example 2 we get

TheOREM 1. Let $S \in L_{(\omega)}^{\left(M_{p}\right) \prime}(\Gamma)$ and $F(z)=\mathcal{L} S(z)$ for $\operatorname{Re} z<\omega$. Then $F \in \mathcal{O}_{v}^{\left(M_{p}\right)}(\operatorname{Re} z<\omega)$.

Theorem 2. Let $\omega_{1} \leq \omega_{2}, S_{1} \in L_{\left(\omega_{1}\right)}^{\left(M_{p}\right) \prime}(\Gamma)$ and $S_{2} \in L_{\left(\omega_{2}\right)}^{\left(M_{p}\right) \prime}(\Gamma)$. If

$$
\mathcal{L} S_{1}(z)=\mathcal{L} S_{2}(z) \quad \text { for } \operatorname{Re} z<\omega_{1}
$$

then $S_{1}=S_{2}$ in $L_{\left(\omega_{1}\right)}^{\left(M_{p}\right) \prime}(\Gamma)$. 
Pro of. We have to prove that for arbitrary $\varphi \in L_{\left(\omega_{1}\right)}^{\left(M_{p}\right)}(\Gamma), S_{1}[\varphi]=S_{2}[\varphi]$. To this end fix $\varphi \in L_{\left(\omega_{1}\right)}^{\left(M_{p}\right)}(\Gamma)$, choose $b<\omega_{1}$ such that $\varphi \in L_{b}^{\left(M_{p}\right)}(\Gamma)$ and take $b<a<\omega_{1}$. Since by (15), $S_{1}\left[\exp _{c}\right]=S_{2}\left[\exp _{c}\right]$ for $c \leq a$ the proof follows from Proposition 1.

To prove the converse of Theorem 1 we need two lemmas. The first one is a restatement of Lemma 9.1 of [11] (cf. also [12]).

Lemma 2. Let $a \in \mathbb{R}$. Suppose that $G$ is holomorphic on the set $\{\operatorname{Re} z \leq b\}$ and satisfies there the estimate

$$
|G(z)| \leq \frac{C}{\langle z\rangle^{2}} e^{v \operatorname{Re} z}
$$

with some $C<\infty, v \in \mathbb{R}$. Put

$$
g(y)=\frac{1}{2 \pi i} \int_{c+i \mathbb{R}} G(z) e^{-z y} d z \quad \text { for } y \in \mathbb{R} .
$$

Then $g$ does not depend on the choice of $c \leq b$; it is a continuous function on $\mathbb{R}$ with support in $\Gamma=[v, \infty)$; the function $\Gamma \ni y \rightarrow e^{b y} g(y)$ is bounded; $g \in L_{(b)}^{\left(M_{p}\right) \prime}(\Gamma)$ and $G(z)=\mathcal{L} g(z)$ for $\operatorname{Re} z<b$.

Lemma 3. Let $\widetilde{\omega} \in \mathbb{R}$ and $k>0$. Then there exists a symbol $P$ of class $\left(M_{p}\right)$ not vanishing on $\{\operatorname{Re} z<\widetilde{\omega}+1\}$ such that

$$
\frac{\exp M(k|z|)}{P(z)} \leq \frac{1}{\langle z\rangle^{2}} \quad \text { for } \operatorname{Re} z \leq \widetilde{\omega} .
$$

Proof. Since $m_{p} \rightarrow \infty$ as $p \rightarrow \infty\left(\right.$ by $\left(\right.$ M. $\left.3^{\prime}\right)$ ) we can find $p_{0} \in \mathbb{N}$ such that $m_{p}>2 k|\widetilde{\omega}|+k$ and $\left|m_{p}-k z\right| \geq k|z|$ for $p \geq p_{0}$ and $\operatorname{Re} z \leq \widetilde{\omega}$. Put

$$
P(z)=(z-\widetilde{\omega}-1)^{p_{0}+1} \prod_{p=p_{0}}^{\infty}\left(1-\frac{k z}{m_{p}}\right) \quad \text { for } z \in \mathbb{C} .
$$

Then $P$ does not vanish on $\{\operatorname{Re} z<\widetilde{\omega}\}$ and by the Hadamard factorization theorem (cf. [2], Propositions 4.5 and 4.6) it is a symbol of class $\left(M_{p}\right)$. On the other hand, if $\operatorname{Re} z \leq \widetilde{\omega}$ we estimate from below:

$$
\begin{aligned}
\left|\prod_{p=p_{0}}^{\infty}\left(1-\frac{k z}{m_{p}}\right)\right| & \geq \prod_{p=p_{0}}^{\infty}\left(1-\frac{k|\widetilde{\omega}|}{m_{p}}\right) \sup _{q \geq p_{0}} \prod_{p=p_{0}}^{q}\left|1-\frac{k z}{m_{p}}\right| \\
& \geq \prod_{p=p_{0}}^{\infty}\left(1-\frac{k|\widetilde{\omega}|}{m_{p}}\right) \sup _{q \geq p_{0}} \prod_{p=p_{0}}^{q} \frac{k|z|}{m_{p}} \\
& =C|z|^{-p_{0}+1} \exp M(k|z|),
\end{aligned}
$$


where

$$
C=\frac{M_{p_{0}-1}}{M_{0}} \prod_{p=p_{0}}^{\infty}\left(1-\frac{k|\widetilde{\omega}|}{m_{p}}\right)>0 .
$$

Hence, possibly multiplying $P$ by a suitable constant, we get (16).

Theorem 3. Let $\omega \in \mathbb{R} \cup\{\infty\}$ and let $F \in \mathcal{O}_{v}^{\left(M_{p}\right)}(\operatorname{Re} z<\omega)$. Then there exists a Laplace ultradistribution $S \in L_{(\omega)}^{\left(M_{p}\right) \prime}(\Gamma)$ such that

$$
F(z)=\mathcal{L} S(z) \quad \text { for } \operatorname{Re} z<\omega .
$$

Proof. Fix $a<\omega$. Choose $\widetilde{\omega} \in \mathbb{R}$ such that $a<\widetilde{\omega}<\omega$ and assume (14). By Lemma 3 we can find a symbol $P$ of class $\left(M_{p}\right)$, not vanishing on $\{\operatorname{Re} z<\widetilde{\omega}+1\}$ and satisfying (16). Next we apply Lemma 2 to the function

$$
G(z)=\frac{F(z)}{P(z)}, \quad \operatorname{Re} z \leq \widetilde{\omega} .
$$

We get a continuous function $g$ which belongs to $L_{(a)}^{\left(M_{p}\right) \prime}(\Gamma)$ and satisfies $\mathcal{L} g(z)=G(z)$ for $\operatorname{Re} z<a$. Put $S=P(-D) g$. Then $S \in L_{(a)}^{\left(M_{p}\right)^{\prime}}(\Gamma)$ and $\mathcal{L} S(z)=P(z) \mathcal{L} g(z)=F(z)$ for $\operatorname{Re} z<a$.

Thus for every $a<\omega$ we can find $S_{a} \in L_{(a)}^{\left(M_{p}\right) \prime}(\Gamma)$ such that $\mathcal{L} S_{a}(z)=$ $F(z)$ for $\operatorname{Re} z<a$. By Theorem 2 the definition $S=S_{a}$ on $L_{(a)}^{\left(M_{p}\right)}(\Gamma), a<\omega$, defines correctly a functional $S \in L_{(\omega)}^{\left(M_{p}\right) \prime}(\Gamma)$ which satisfies (17).

It follows from the proof of Theorem 3 that Laplace ultradistributions can be characterized as follows.

TheOREM 4 (Structure theorem). An ultradistribution $S \in D^{\left(M_{p}\right) \prime}(\mathbb{R})$ is in $L_{(\omega)}^{\left(M_{p}\right) \prime}(\Gamma)$ if and only if for every a $<\omega$ there exist an ultradifferential operator $P_{a}$ of class $\left(M_{p}\right)$ and a function $g_{a}$ continuous on $\mathbb{R}$ with support in $\Gamma$ such that

and

$$
\begin{aligned}
\left|g_{a}(y)\right| & \leq C e^{-a y} & & \text { for } y \in \Gamma, \\
\left|\mathcal{L} g_{a}(z)\right| & \leq \frac{C}{\langle z\rangle^{2}} e^{v \operatorname{Re} z} & & \text { for } \operatorname{Re} z<a
\end{aligned}
$$

$$
S=P_{a}(D) g_{a} \quad \text { in } L_{(a)}^{\left(M_{p}\right) \prime}(\Gamma) .
$$

3. Boundary value representation. In this section we use the following version of the Phragmén-Lindelöf theorem.

3-Line theorem ([1]). Let $R>0$ and $F \in \mathcal{O}\left(\Gamma_{R}\right) \cap C^{0}\left(\bar{\Gamma}_{R}\right)$. Suppose that for some $k>0$ the function

$$
\bar{\Gamma}_{R} \ni z \rightarrow \exp \{-k|z|\} F(z)
$$


is bounded. If $F$ is bounded on the boundary of $\bar{\Gamma}_{R}$ then it is also bounded on $\Gamma_{R}$.

Definition. Let $R>0, k>0$ and $a \in \mathbb{R}$. We define the spaces

$$
\widetilde{L}_{a}\left(\Gamma_{R}\right)=\left\{\varphi \in \mathcal{O}\left(\Gamma_{R}\right) \cap C^{0}\left(\bar{\Gamma}_{R}\right):\|\varphi\|_{a, \Gamma_{R}}=\sup _{z \in \bar{\Gamma}_{R}}\left|\varphi(z) e^{a z}\right|<\infty\right\},
$$

$\widetilde{L}_{a, k}^{\left(M_{p}\right)}\left(\Gamma_{R} \backslash \Gamma\right)=\left\{\varphi \in \mathcal{O}\left(\Gamma_{R} \backslash \Gamma\right): \varphi \cdot \exp \left\{-M^{*}(k /|\operatorname{Im} z|)\right\} \in C^{0}\left(\bar{\Gamma}_{R}\right)\right.$,

$$
\left.\|\varphi\|_{a, k, \Gamma_{R}}^{\left(M_{p}\right)}=\sup _{z \in \bar{\Gamma}_{R}}\left|\varphi(z) \exp \left\{a z-M^{*}(k /|\operatorname{Im} z|)\right\}\right|<\infty\right\} .
$$

By the 3 -line theorem $\widetilde{L}_{a}\left(\Gamma_{R}\right)$ is a closed subspace of the Banach space $\widetilde{L}_{a, k}^{\left(M_{p}\right)}\left(\Gamma_{R} \backslash \Gamma\right)$ and we can define

$$
H_{a, k}^{\left(M_{p}\right)}\left(\Gamma_{R}, \Gamma\right)=\widetilde{L}_{a, k}^{\left(M_{p}\right)}\left(\Gamma_{R} \backslash \Gamma\right) / \widetilde{L}_{a}\left(\Gamma_{R}\right) .
$$

Further, we define

$$
\begin{gathered}
\widetilde{L}_{a}(\mathbb{C})=\lim _{R \rightarrow \infty} \widetilde{L}_{a}\left(\Gamma_{R}\right), \quad \widetilde{L}_{a, k}^{\left(M_{p}\right)}(\mathbb{C} \backslash \Gamma)={\underset{l}{R \rightarrow \infty}}_{\lim _{a, k}} \widetilde{L}_{a, k}^{\left(M_{p}\right)}\left(\Gamma_{R} \backslash \Gamma\right), \\
\widetilde{L}_{a}^{\left(M_{p}\right)}(\mathbb{C} \backslash \Gamma)=\varliminf_{k \rightarrow \infty} \widetilde{L}_{a, k}^{\left(M_{p}\right)}(\mathbb{C} \backslash \Gamma), \\
\widetilde{H}_{a}^{\left(M_{p}\right)}(\mathbb{C}, \Gamma)=\widetilde{L}_{a}^{\left(M_{p}\right)}(\mathbb{C} \backslash \Gamma) / \widetilde{L}_{a}(\mathbb{C}), \quad \underset{\sim}{H_{a}^{\left(M_{p}\right)}}(\Gamma)=\lim _{\substack{R \rightarrow 0 \\
k \rightarrow \infty}} H_{a, k}^{\left(M_{p}\right)}\left(\Gamma_{R}, \Gamma\right) .
\end{gathered}
$$

Let $a<b$. Then the natural mappings

$$
\widetilde{H}_{b}^{\left(M_{p}\right)}(\mathbb{C}, \Gamma) \rightarrow \widetilde{H}_{a}^{\left(M_{p}\right)}(\mathbb{C}, \Gamma), \quad \underset{\sim}{H_{b}^{\left(M_{p}\right)}}(\Gamma) \rightarrow \underset{\sim}{H_{a}^{\left(M_{p}\right)}}(\Gamma)
$$

are well defined and by the 3-line theorem they are injections. Thus, for $\omega \in \mathbb{R} \cup\{\infty\}$, we can define

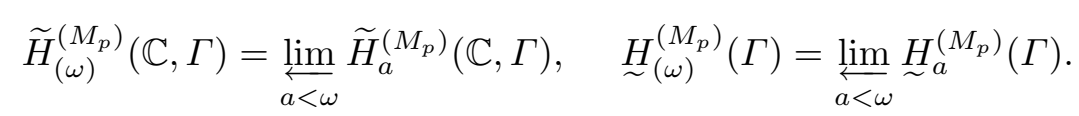

An element $f \in \widetilde{H}_{(\omega)}^{\left(M_{p}\right)}(\mathbb{C}, \Gamma)$ is given by a set $\left\{F_{a}\right\}_{a<\omega}$ of functions such that for $a<\omega, F_{a} \in \widetilde{L}_{a}^{\left(M_{p}\right)}(\mathbb{C} \backslash \Gamma)$ and for $a<b<\omega, F_{a}-F_{b} \in \widetilde{L}_{a}(\mathbb{C})$. On the other hand, an element $g \in \underset{\sim}{H_{(\omega)}^{\left(M_{p}\right)}}(\Gamma)$ is given by a set $\left\{G_{a}\right\}_{a<\omega}$ of functions such that for $a<\omega$ there exist $R_{a}>0$ and $k_{a}<\infty$ such that $G_{a} \in \widetilde{L}_{a, k_{a}}^{\left(M_{p}\right)}\left(\Gamma_{R_{a}} \backslash \Gamma\right)$ and for $a<b<\omega, G_{a}-G_{b} \in \widetilde{L}_{a}\left(\Gamma_{R_{a}} \cap \Gamma_{R_{b}}, \Gamma\right)$.

The natural mapping

$$
i: \widetilde{H}_{(\omega)}^{\left(M_{p}\right)}(\mathbb{C}, \Gamma) \rightarrow \underset{\sim}{H}(\omega)(\Gamma)
$$

is defined by retaining the same set of defining functions. Obviously it is an injection. 
Lemma 4. Let $S \in L_{(\omega)}^{\left(M_{p}\right) \prime}(\Gamma)$ and $a<\omega$. Put

$$
\mathcal{C}_{a} S(z)=\frac{1}{2 \pi i} S\left[\frac{e^{a(\cdot-z)}}{z-\cdot}\right] \quad \text { for } z \in \mathbb{C} \backslash \Gamma .
$$

Then $\mathcal{C}_{a} S \in \widetilde{L}_{a}^{\left(M_{p}\right)}(\mathbb{C} \backslash \Gamma)$. Furthermore, if $a<b<\omega$ then $\mathcal{C}_{a} S-\mathcal{C}_{b} S \in$ $\widetilde{L}_{a}(\mathbb{C})$.

Pr o of. Take $a<c<\omega$. By Theorem 4 we can find an ultradifferential operator $P_{c}$ of class $\left(M_{p}\right)$ and a continuous function $g_{c}$ with support in $\Gamma$ satisfying $\left|g_{c}(y)\right| \leq C e^{-c y}$ for $y \in \Gamma$ and $S=P_{c}(D) g_{c}$ in $L_{(c)}^{\left(M_{p}\right) \prime}(\Gamma)$. Since for fixed $z \in \mathbb{C} \backslash \Gamma$ the function

$$
\Gamma \ni y \rightarrow \frac{e^{a(y-z)}}{z-y}
$$

belongs to $L_{(c)}^{\left(M_{p}\right)}(\Gamma)$ we have

$$
\mathcal{C}_{a}(z)=\frac{1}{2 \pi i} e^{-a z} \int_{\Gamma} g_{c}(y) P_{c}^{*}\left(D_{y}\right)\left(\frac{e^{a y}}{z-y}\right) d y .
$$

Let $P_{c}^{*}(D)=\sum_{\alpha=0}^{\infty}(-1)^{\alpha} a_{\alpha} D^{\alpha}$ with $a_{\alpha}$ satisfying (5) and let $R>0$. Then for $z \in \Gamma_{R} \backslash \Gamma$ we estimate

$$
\begin{aligned}
\left|P_{c}^{*}(D)\left(\frac{e^{a y}}{z-y}\right)\right| & \leq \sum_{\alpha=0}^{\infty}\left|a_{\alpha}\right|\left|D^{\alpha}\left(\frac{e^{a y}}{z-y}\right)\right| \leq e^{a y} \sum_{\beta=0}^{\infty} \frac{|a|^{\beta}}{\beta !} \sum_{\alpha=\beta}^{\infty} \frac{\left|a_{\alpha}\right| \alpha !}{|\operatorname{Im} z|^{\alpha-\beta+1}} \\
& \leq e^{a y} \sum_{\beta=0}^{\infty} \frac{|a|^{\beta} R^{\beta}}{\beta !} \sum_{\alpha=0}^{\infty} \frac{\left|a_{\alpha}\right| \alpha !}{|\operatorname{Im} z|^{\alpha+1}} \\
& \leq e^{a y+|a| R} \cdot \frac{A C}{H K} \exp M^{*}\left(\frac{2 H K}{|\operatorname{Im} z|}\right)
\end{aligned}
$$

since by (5) and (M.2') we have

$$
\begin{aligned}
\sum_{\alpha=0}^{\infty} \frac{\left|a_{\alpha}\right| \alpha !}{|\operatorname{Im} z|^{\alpha+1}} & \leq C \sum_{\alpha=0}^{\infty} \frac{K^{\alpha} \alpha !}{|\operatorname{Im} z|^{\alpha+1} M_{\alpha}} \leq 2 C \sup _{\alpha \in \mathbb{N}_{0}} \frac{(2 K)^{\alpha} \alpha !}{|\operatorname{Im} z|^{\alpha+1} M_{\alpha}} \\
& \leq \frac{2 A C}{2 H K} \sup _{\alpha \in \mathbb{N}_{0}} \frac{(2 H K)^{\alpha+1} \alpha !}{|\operatorname{Im} z|^{\alpha+1} M_{\alpha+1}} \leq \frac{A C}{H K} \exp M^{*}\left(\frac{2 H K}{|\operatorname{Im} z|}\right)
\end{aligned}
$$

Put $k=2 H K$. Then for every $R>0$ there exists $C<\infty$ such that

$$
\left|\mathcal{C}_{a} S(z)\right| \leq C \exp \left\{-a \operatorname{Re} z+M^{*}(k /|\operatorname{Im} z|)\right\} \quad \text { for } z \in \Gamma_{R} \backslash \Gamma \text {. }
$$

Thus, $\mathcal{C}_{a} S \in \widetilde{L}_{a}^{\left(M_{p}\right)}(\mathbb{C} \backslash \Gamma)$. If $a<b<\omega$ we take $c<\omega$ such that $b<c$ and 
note that for $z \in \mathbb{C}$ the function

$$
\Gamma \ni y \rightarrow \frac{e^{a(y-z)}-e^{b(y-z)}}{z-y}
$$

belongs to $L_{(c)}^{\left(M_{p}\right)}(\Gamma)$. Thus, the holomorphic extension of $\mathcal{C}_{a} S-\mathcal{C}_{b} S$ is given by

$$
\left(\mathcal{C}_{a} S-\mathcal{C}_{b} S\right)(z)=\frac{1}{2 \pi i} \int_{\Gamma} g_{c}(y) P_{c}^{*}(D)\left(\frac{e^{a(y-z)}-e^{b(y-z)}}{z-y}\right) d y
$$

and we easily find that $\mathcal{C}_{a}-\mathcal{C}_{b} \in \widetilde{L}_{a}(\mathbb{C})$.

Definition. Let $S \in L_{(\omega)}^{\left(M_{p}\right) \prime}(\Gamma)$. Then by Lemma 4 the set $\left\{\mathcal{C}_{a} S\right\}_{a<\omega}$ of functions defines an element $f \in \widetilde{H}_{(\omega)}^{\left(M_{p}\right)}(\Gamma)$. We call $f$ the Cauchy transform of $S$ and write $f=\mathcal{C} S$.

Proposition 2. Let $F_{a} \in \widetilde{L}_{a, k}^{\left(M_{p}\right)}\left(\Gamma_{R} \backslash \Gamma\right)$ with $a \leq 0$. Then there exist an ultradifferential operator $P_{a}(D)$ of class $\left(M_{p}\right)$ and functions $H_{a}^{ \pm} \in \mathcal{O}\left(\Gamma_{R} \cap\right.$ $\{ \pm \operatorname{Im} z>0$ or $\operatorname{Re} z<v\})$ such that

$1^{\circ} P_{a}(D) H_{a}^{ \pm}=F_{a}$;

$2^{\circ}$ For every $0<R^{\prime}<R$ and $a^{\prime}<a$ there exists $C<\infty$ such that

$$
\left|H_{a}^{ \pm}(z)\right| \leq C \exp \left\{-a^{\prime} \operatorname{Re} z\right\} \quad \text { for } z \in \Gamma_{R^{\prime}} \cap\{ \pm \operatorname{Im} z>0\} ;
$$

$3^{\circ} H_{a}^{ \pm}(x+i y)$ converges uniformly as $y \rightarrow 0+$ to a function $h_{a}^{ \pm}$continuous on $(v-R, \infty)$ and analytic on $(v-R, v)$ satisfying

$$
\left|h_{a}^{ \pm}(x)\right| \leq C \exp \left\{-a^{\prime} x\right\} \quad \text { for } x \in\left(v-R^{\prime}, \infty\right) .
$$

Furthermore, if we put

$$
S_{a}=F_{a}^{+}(x+i 0)-F_{a}^{-}(x-i 0), \quad \text { where } F_{a}^{ \pm}(x \pm i 0)=P_{a}(D) h_{a}^{ \pm},
$$

then $S_{a}$ extends to a Laplace ultradistribution $\widetilde{S}_{a} \in L_{(a)}^{\left(M_{p}\right) \prime}(\Gamma)$ defined by

(20) $\quad \widetilde{S}_{a}[\varphi]=\int_{v-R}^{\infty}\left(h_{a}^{+}(x)-h_{a}^{-}(x)\right) P_{a}^{*}(D) \mathcal{E} \varphi(x) d x \quad$ for $\varphi \in L_{(a)}^{\left(M_{p}\right)}(\Gamma)$.

In $(20), \mathcal{E}$ is a linear continuous extension mapping $\mathcal{E}: L_{(a)}^{\left(M_{p}\right)}(\Gamma) \rightarrow$ $L_{(a)}^{\left(M_{p}\right)}([v-R, \infty))$, which exists by the Seeley extension theorem.

Proof. Put

$$
P(\zeta)=(1+\zeta)^{2} \prod_{p=1}^{\infty}\left(1+\frac{k \zeta}{m_{p}}\right) \quad \text { for } \zeta \in \mathbb{C} .
$$


Then $P$ is a symbol of class $\left(M_{p}\right)$. Define the Green kernel for $P$ by

$$
G(z)=\frac{1}{2 \pi i} \int_{0}^{\infty} \frac{e^{z \zeta}}{P(\zeta)} d \zeta \quad \text { for } \operatorname{Re} z<0
$$

Then by Lemma 11.4 of [2], $G$ can be holomorphically continued to the Riemann domain $\{z:-\pi / 2<\arg z<5 \pi / 2\}$, on which we have

$$
P(D) G(z)=-\frac{1}{2 \pi i} \frac{1}{z} \text {. }
$$

Furthermore, since for any $0 \leq \psi<\pi / 2$,

$$
\frac{|z|}{|1-z|} \leq \frac{1}{\cos \psi} \text { and } \quad \frac{1}{|1-z|} \leq \frac{1}{\cos \psi}
$$

for $z \in \mathbb{C}$ with $|\arg z| \leq \psi+\pi / 2$, following the proof of the above-mentioned lemma we conclude that on the domain $\{-\psi \leq \arg z \leq 2 \pi+\psi\}$, $G$ is bounded by $C / \cos \psi$ with $C<\infty$ not depending on $\psi$. We also have

$$
|g(x)| \leq A \sqrt{x} \exp \left\{-M^{*}(k / x)\right\} \quad \text { for } x>0,
$$

where

$$
g(z)=G_{+}(z)-G_{-}\left(e^{2 \pi i} z\right) \quad \text { for } \operatorname{Re} z>0,
$$

$G_{+}$being the branch of $G$ on $\{-\pi / 2<\arg z<\pi / 2\}$ and $G_{-}$that on $\{3 \pi / 2<\arg z<5 \pi / 2\}$. Put

$$
H_{a}^{ \pm}(z)= \pm i \int_{\gamma_{ \pm}} G( \pm i(z-w)) F_{a}(w) d w
$$

where $\gamma_{ \pm}$is a closed curve encircling $z$ once, in the anticlockwise direction, such that $-\pi / 2<\arg ( \pm i(z-w))<5 \pi / 2$ for $w \in \gamma_{ \pm}$. We choose a starting point $\stackrel{\circ}{z}_{ \pm}$of $\gamma_{ \pm}$in such a way that $\left|\arg \left\{ \pm i\left(z-\stackrel{\circ}{z}_{ \pm}\right)\right\}\right|<\pi / 2$. Then $H_{a}^{ \pm}$is a holomorphic function on $\Gamma_{R} \cap\{ \pm \operatorname{Im} z>0$ or $\operatorname{Re} z<v\}$ and does not depend on the choice of $\gamma_{ \pm}$with a fixed starting point $\stackrel{\circ}{z}_{ \pm}$. For a fixed $\gamma$ and $z$ changing in a compact set in the domain bounded by $\gamma$ we have

$$
\begin{aligned}
P(D) H_{a}^{ \pm}(z) & = \pm i \int_{\gamma} P\left(D_{z}\right) G( \pm i(z-w)) F_{a}(w) d w \\
& =\frac{-1}{2 \pi i} \int_{\gamma} \frac{F_{a}(w)}{z-w} d w=F_{a}(z) .
\end{aligned}
$$

Let $0<R^{\prime}<R$ and $z \in \Gamma_{R^{\prime}} \cap\{\operatorname{Im} z>0\}$. Fix $\stackrel{\circ}{z} \in \Gamma_{R} \cap\left\{\operatorname{Im} z>r^{\prime}\right\} \cap\{\operatorname{Re} z<$ $\left.v-R^{\prime}\right\}$ and take $\gamma_{+}=\gamma_{1} \cup \gamma_{2} \cup \gamma_{3} \cup \gamma_{4}$, where $\gamma_{1}=[\stackrel{\circ}{x}+i \grave{y}, x+i \grave{y}]$, $\gamma_{2}=[x+i \stackrel{\circ}{y}, x+i y], \gamma_{3}=[x+i y, x+i \stackrel{\circ}{y}]$ and $\gamma_{4}=[x+i \stackrel{\circ}{y}, \stackrel{\circ}{x}+i \stackrel{\circ}{y}]$. Since $0 \leq \arg (i(z-w)) \leq \psi$ for $w \in \gamma_{1}$ and $2 \pi \leq \arg (i(z-w)) \leq 2 \pi+\psi$ for $w \in \gamma_{4}$, where $0 \leq \psi<\pi / 2$ is such that $\tan \psi=(x-\stackrel{\circ}{x}) /(\stackrel{\circ}{y}-y)$, by the 
boundedness of $G$ on $\{0 \leq \arg \psi \leq 2 \pi+\psi\}$ we have $|G(i(z-w))| \leq C x$ for $w \in \gamma_{1} \cup \gamma_{4}$, where $C$ does not depend on $x$. So

$$
\left|\int_{\gamma_{1} \cup \gamma_{4}} G(i(z-w)) F_{a}(w) d w\right| \leq C x^{2} e^{-a x} \quad \text { for } z \in \Gamma_{R^{\prime}} \cap\{\operatorname{Im} z>0\} .
$$

On the other hand,

$$
\begin{aligned}
\mid \int_{\gamma_{2} \cup \gamma_{3}} G(i(z-w)) & F_{a}(w) d w|=|-i \int_{0}^{\grave{y}-y} g(t) F(x+i(y+t)) d t \mid \\
\leq & A C \int_{0}^{\grave{y}-y} \sqrt{t} \exp \left\{M^{*}\left(\frac{k}{y+t}\right)-M^{*}\left(\frac{k}{t}\right)-a x\right\} d t \\
\leq & A C(\stackrel{\circ}{y}-y)^{3 / 2} e^{-a x} \quad \text { for } z \in \Gamma_{R^{\prime}} \cap\{\operatorname{Im} z>0\} .
\end{aligned}
$$

Thus, for any $a^{\prime}<a$ one can find $C<\infty$ such that

$$
\left|H_{a}^{+}(z)\right| \leq C e^{-a^{\prime} x} \quad \text { for } z \in \Gamma_{R^{\prime}} \cap\{\operatorname{Im} z>0\} .
$$

The estimate of $H_{a}^{-}$is obtained in an analogous way.

The assertion $3^{\circ}$ is clear from the above estimates.

Let $\psi \in D^{\left(M_{p}\right)}((v-R, \infty))$. By $1^{\circ}$ and $2^{\circ}$ we derive

$$
\begin{aligned}
F_{a}(x \pm i 0)[\psi] & =P_{a}(D) h_{a}^{ \pm}[\psi]=\int_{v-R}^{\infty} h_{a}^{ \pm}(x) P_{a}^{*}(D) \psi(x) d x \\
& =\lim _{y \rightarrow 0+} \int_{v-R}^{\infty} H_{a}(x \pm i y) P_{a}^{*}(D) \psi(x) d x \\
& =\lim _{y \rightarrow 0+} \int_{v-R}^{\infty} F_{a}(x \pm i y) \psi(x) d x .
\end{aligned}
$$

Since for $\psi \in D^{\left(M_{p}\right)}((v-R, v))$,

$$
S_{a}[\psi]=\lim _{y \rightarrow 0+} \int_{v-R}^{v} P_{a}(D)\left(H_{a}^{+}(x+i y)-H_{a}^{-}(x-i y)\right) \psi(x) d x=0,
$$

$S_{a}$ has support in $\Gamma$ and we can define the extension of $S_{a}$ by (20).

Let $f \in \underset{\sim}{H_{a}^{\left(M_{p}\right)}}(\Gamma)$. Then there exist $R>0, k<\infty$ and a function $F_{a} \in \widetilde{L}_{a, k}^{\left(M_{p}\right)}\left(\widetilde{\Gamma_{R}} \backslash \Gamma\right)$ such that $f=\left[F_{a}\right]$. If $a \leq 0$ we can apply Proposition 2 to $F_{a}$. If $a>0$ then we apply Proposition 2 to $F_{a}^{*}=e^{a z} F_{a}$ instead of $F_{a}$. In this case denote by $\widetilde{S}_{a}^{*}$ the element of $L_{(0)}^{\left(M_{p}\right) \prime}(\Gamma)$ given by (20) and define $\widetilde{S}_{a}=e^{-a x} \widetilde{S}_{a}^{*}$. In both cases $\widetilde{S}_{a} \in L_{(a)}^{\left(M_{p}\right) \prime}(\Gamma)$ does not depend on the choice of a defining function $F_{a}$ for $f$. Thus, the assignment $f \rightarrow \widetilde{S}_{a}$ defines a 
mapping

$$
b: \underset{\sim}{H_{a}^{\left(M_{p}\right)}}(\Gamma) \rightarrow L_{(a)}^{\left(M_{p}\right) \prime}(\Gamma) .
$$

Since (21) holds for every $a<\omega$ we have

$$
b: \underset{\sim}{H_{(\omega)}^{\left(M_{p}\right)}} \rightarrow \lim _{a<\omega} L_{(a)}^{\left(M_{p}\right) \prime}(\Gamma) \simeq\left(\lim _{a<\omega} L_{(a)}^{\left(M_{p}\right)}(\Gamma)\right)^{\prime}=L_{(\omega)}^{\left(M_{p}\right) \prime}(\Gamma),
$$

where the isomorphism $\simeq$ follows by the formula (1.2) of [2].

TheOREM 5. The mapping

$$
\mathcal{C}: L_{(\omega)}^{\left(M_{p}\right)^{\prime}}(\Gamma) \rightarrow \widetilde{H}_{(\omega)}^{\left(M_{p}\right)}(\mathbb{C}, \Gamma)
$$

is a topological isomorphism with inverse $b \circ i$.

Proof. Let $S \in L_{(\omega)}^{\left(M_{p}\right) \prime}(\Gamma)$ and $\mathcal{C} S=f \in \widetilde{H}_{(\omega)}^{\left(M_{p}\right)}(\Gamma)$. Then

$$
f=\left[\left\{F_{a}\right\}_{a<\omega}\right] \quad \text { with } \quad F_{a}(z)=\frac{1}{2 \pi i} S\left[\frac{e^{a(\cdot-z)}}{z-\cdot}\right] \quad \text { for } z \in \mathbb{C} \backslash \Gamma .
$$

Treat $f$ as an element of $\underset{\sim}{H_{(\omega)}^{\left(M_{p}\right)}}(\Gamma)$ and put $\widetilde{S}=b(f) \in L_{(\omega)}^{\left(M_{p}\right) \prime}(\Gamma)$. Fix $a<\omega$. Then for $\varphi \in Y_{(a)}$ we have

$$
\widetilde{S}[\varphi]=-\int_{\partial \Gamma_{\varepsilon}} F_{a}(z) \varphi(z) d z=S\left[-\frac{1}{2 \pi i} \int_{\partial \Gamma_{\varepsilon}} \frac{e^{a(\cdot-z)}}{z-\cdot} \varphi(z) d z\right]=S[\varphi],
$$

by the Cauchy integral formula. Since $Y_{(a)}$ is dense in $L_{(a)}^{\left(M_{p}\right)}(\Gamma)$ and $a<\omega$ is arbitrary, we have $\widetilde{S}[\varphi]=S[\varphi]$ for $\varphi \in L_{(\omega)}^{\left(M_{p}\right)}(\Gamma)$. Thus $b \circ i \circ \mathcal{C}=\mathrm{id}$.

Let $f \in \widetilde{H}_{(\omega)}^{\left(M_{p}\right)}(\mathbb{C}, \Gamma), f=\left[\left\{F_{a}\right\}_{a<\omega}\right]$ with $F_{a} \in \widetilde{L}_{a}^{\left(M_{p}\right)}(\mathbb{C} \backslash \Gamma)$ and $F_{a}-$ $F_{b} \in \widetilde{L}_{a}(\mathbb{C})$ for $a<b<\omega$. Put $\underset{\sim}{f}=i(f)$ and fix $a<\omega$. Then $\underset{\sim}{f}=\left[F_{a}\right]$ in ${\underset{\sim}{H}}_{a}^{\left(M_{p}\right)}(\Gamma)$ and by $(21), \widetilde{S}_{a}=b(\underset{\sim}{\tilde{f}}) \in L_{(a)}^{\left(M_{p}\right) \prime}(\Gamma)$. Observe that for $\varepsilon>0$ we have

$$
\widetilde{S}_{a}[\varphi]=-\int_{\partial \Gamma_{\varepsilon}} F_{a}(z) \varphi(z) d z \quad \text { for } \varphi \in Y_{(a)} .
$$

On the other hand, by the part of the theorem just proved,

$$
\widetilde{S}_{a}[\varphi]=-\int_{\partial \Gamma_{\varepsilon}} \frac{1}{2 \pi i} \widetilde{S}_{a}\left[\frac{e^{a(\cdot-z)}}{z-\cdot}\right] \varphi(z) d z \quad \text { for } \varphi \in Y_{(a)} .
$$

So for $\varphi \in Y_{(a)}$,

(22) $\int_{\partial \Gamma_{\varepsilon}} \psi_{a}(z) \varphi(z) d z=0, \quad$ where $\quad \psi_{a}(z)=\frac{1}{2 \pi i} \widetilde{S}_{a}\left[\frac{e^{a(\cdot-z)}}{z-\cdot}\right]-F_{a}(z)$. 
Then $\psi_{a} \in \widetilde{L}_{a, k}^{\left(M_{p}\right)}(\mathbb{C} \backslash \Gamma)$ and we shall show that $\psi_{a}$ extends holomorphically to a function $\widetilde{\psi}_{a} \in \widetilde{L}_{a}(\mathbb{C})$, which proves that $\mathcal{C} \circ b \circ i=\mathrm{id}$. To this end observe that $(22)$ holds also for $\varphi \in L_{(a)}^{\left(M_{p}\right)}(\Gamma) \cap \mathcal{O}\left(\Gamma_{\varepsilon}\right)$ and put for any $b<a, R>\varepsilon$,

$$
G_{b}(z)=\int_{\partial \Gamma_{R}} \psi_{a}(\zeta) \frac{e^{b(\zeta-z)}}{z-\zeta} d \zeta \quad \text { for } z \in \Gamma_{R}
$$

Then $\left|G_{b}(z)\right| \leq C \exp \{-b \operatorname{Re} z\}$ for $z \in \bar{\Gamma}_{R^{\prime}}$ with $R^{\prime}<R$. Using (22) with $\varphi(\zeta)=\exp \{b(\zeta-z)\} /(z-\zeta), z \in \Gamma_{R} \backslash \bar{\Gamma}_{\varepsilon}$, we get $G_{b}(z)=\psi_{a}(z)$ for $z \in \Gamma_{R} \backslash \bar{\Gamma}_{\varepsilon}$. Put

$$
\widetilde{\psi}_{a}(z)= \begin{cases}\psi_{a}(z) & \text { for } z \in \Gamma_{R} \backslash \Gamma \\ G_{b}(z) & \text { for } z \in \Gamma_{R}\end{cases}
$$

Then $\widetilde{\psi}_{a} \in \mathcal{O}\left(\Gamma_{R}\right)$ and by the 3 -line theorem $\widetilde{\psi}_{a} \in \widetilde{L}_{a}\left(\Gamma_{R}\right)$. Since $R>\varepsilon$ was arbitrary we have $\widetilde{\psi} \in \widetilde{L}_{a}(\mathbb{C})$.

\section{Mellin ultradistributions}

Definition. Let $\omega \in \mathbb{R} \cup\{\infty\}, v \in \mathbb{R}$ and $I=\left(0, e^{-v}\right]$. We define the space $M_{(\omega)}^{\left(M_{p}\right) \prime}(I)$ of Mellin ultradistributions as the dual space of

$$
M_{(\omega)}^{\left(M_{p}\right)}(I)=\lim _{a<\omega} \varliminf_{h>0} M_{a, h}^{\left(M_{p}\right)}(I),
$$

where for any $a \in \mathbb{R}$ and $h>0$,

$$
M_{a, h}^{\left(M_{p}\right)}(I)=\left\{\psi \in C^{\infty}(I): \varrho_{a, h}^{\left(M_{p}\right)}(\psi)=\sup _{x \in I} \sup _{\alpha \in \mathbb{N}_{0}} \frac{\left|x^{a+1}(D x)^{\alpha} \psi(x)\right|}{h^{\alpha} M_{\alpha}}<\infty\right\} .
$$

LEMmA 5. Let $a \in \mathbb{R}, h>0, \psi \in M_{a, h}^{\left(M_{p}\right)}(I)$ and $\varphi=\mu \cdot \psi \circ \mu$. Then $\varphi \in L_{a, h}^{\left(M_{p}\right)}(\Gamma)$ and $\|\varphi\|_{a, h}^{\left(M_{p}\right)}=\varrho_{a, h}^{\left(M_{p}\right)}(\psi)$. Thus, the mapping

$$
M_{(\omega)}^{\left(M_{p}\right)}(I) \ni \psi \rightarrow \mu \cdot \psi \circ \mu \in L_{(\omega)}^{\left(M_{p}\right)}(\Gamma)
$$

is a continuous isomorphism with inverse

$$
L_{(\omega)}^{\left(M_{p}\right)}(\Gamma) \ni \varphi \rightarrow \exp _{1} \circ \mu^{-1} \cdot \varphi \circ \mu^{-1} \in M_{(\omega)}^{\left(M_{p}\right)}(I) .
$$

Proof. The proof follows easily from the formula

$$
D_{y}^{\alpha}(\mu(y) \psi \circ \mu(y))=(-1)^{\alpha} x\left(D_{x} x\right)^{\alpha} \psi(x), \quad \text { for } \alpha \in \mathbb{N}_{0}, x=\mu(y),
$$

which can be proved by induction.

Let $S \in L_{(\omega)}^{\left(M_{p}\right) \prime}(\Gamma)$. Put

$$
S \circ \mu^{-1}[\psi]=S[\mu \cdot \psi \circ \mu] \quad \text { for } \psi \in M_{(\omega)}^{\left(M_{p}\right)}(I) .
$$


Then by Lemma $5, S \circ \mu^{-1}$ is a well defined element of $M_{(\omega)}^{\left(M_{p}\right) \prime}(I)$ and the mapping

is continuous.

$$
L_{(\omega)}^{\left(M_{p}\right) \prime}(\Gamma) \ni S \rightarrow S \circ \mu^{-1} \in M_{(\omega)}^{\left(M_{p}\right) \prime}(I)
$$

Observe that the function

$$
I \ni x \rightarrow x^{-z-1}=\exp _{z+1} \circ \mu^{-1}(x)
$$

belongs to $M_{(\omega)}^{\left(M_{p}\right)}(I)$ if and only if $\operatorname{Re} z<\omega$. Thus, we can define the Mellin transform of $T \in M_{(\omega)}^{\left(M_{p}\right) \prime}(I)$ by

$$
\mathcal{M T}(z)=T\left[\exp _{z+1} \circ \mu^{-1}\right] \quad \text { for } \operatorname{Re} z<\omega .
$$

Let $S \in L_{(\omega)}^{\left(M_{p}\right) \prime}(\Gamma)$ and $T=S \circ \mu^{-1}$. Then for $\operatorname{Re} z<\omega$ we have

$$
\mathcal{M T}(z)=S \circ \mu^{-1}\left[\exp _{z+1} \circ \mu^{-1}\right]=S\left[\exp _{z}\right]=\mathcal{L} S(z) .
$$

\section{Strong quasi-analyticity principle} by

Definition. Let $S \in L_{(\omega)}^{\left(M_{p}\right) \prime}(\Gamma)$. We define the Taylor transform of $S$

We also define

$$
\mathcal{T} S(x)=\mathcal{L} S(\ln x) \quad \text { for } x \in \widetilde{B}\left(e^{\omega}\right)
$$

$$
\begin{aligned}
& \mathcal{O}_{v}^{\left(M_{p}\right)}\left(\widetilde{B}\left(e^{\omega}\right)\right) \\
& \quad=\left\{u \in \mathcal{O}\left(\widetilde{B}\left(e^{\omega}\right)\right):\right.
\end{aligned}
$$

for every $t<e^{\omega}$ there exist $k<\infty$ and $C<\infty$ such that

$$
\left.|u(x)| \leq C \exp \{M(k(\omega-\ln |x|+|\arg x|))\} \cdot|x|^{v} \text { for }|x| \leq t\right\} .
$$

By Theorems 1 and 3 we get

THEOREM 6. The Taylor transformation is an isomorphism of $L_{(\omega)}^{\left(M_{p}\right) \prime}(\Gamma)$ onto $\mathcal{O}_{v}^{\left(M_{p}\right)}\left(\widetilde{B}\left(e^{\omega}\right)\right)$.

Let $u \in \mathcal{O}_{v}^{\left(M_{p}\right)}\left(\widetilde{B}\left(e^{\omega}\right)\right)$. Then for any $t<e^{\omega}, u_{\mid(0, t]} \in M_{(v)}^{\left(M_{p}\right)^{\prime}}((0, t])$ and

$$
\mathcal{M}_{t} u(z)=\int_{0}^{t} u(x) x^{-z-1} d x \quad \text { for } \operatorname{Re} z<v .
$$

By Theorem $6, u(x)=S\left[x^{\cdot}\right]$ for $x \in \widetilde{B}\left(e^{\omega}\right)$ with $S=\mathcal{T}^{-1} u \in L_{(\omega)}^{\left(M_{p}\right) \prime}(\Gamma)$, $\Gamma=[v, \infty)$. For $\operatorname{Re} z<v$ we derive

$$
\mathcal{M}_{t} u(z)=S\left[\int_{0}^{t} x^{-z-1} d x\right]=S\left[\frac{t^{\cdot-z}}{\cdot-z}\right]=-2 \pi i \mathcal{C}_{\ln t} S(z) .
$$


Thus, by Lemma $4, \mathcal{M}_{t} u$ extends holomorphically to a function $\mathcal{M}_{t} u \in$ $\widetilde{L}_{\ln t}^{\left(M_{p}\right)}(\mathbb{C} \backslash \Gamma)$ and the set of functions $\left\{\mathcal{M}_{t} u\right\}_{t<e^{\omega}}$ defines an element of $\widetilde{H}_{(\omega)}^{\left(M_{p}\right)}(\mathbb{C}, \Gamma)$, which will be denoted by $\mathcal{M} u$ and called the Mellin transform of $u$.

We can summarize Theorems 1, 3, 5 and 6 as follows:

COROLlary 2. We have the following diagram of linear topological isomorphisms:

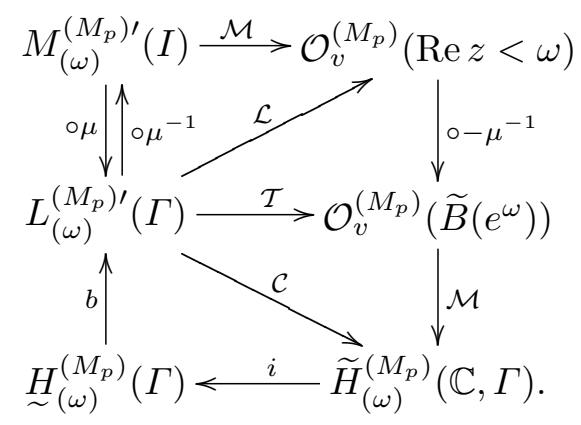

Following [13] we call the elements of $\mathcal{O}_{v}^{\left(M_{p}\right)}(\operatorname{Re} z<\omega)$ generalized analytic functions determined by $L_{(\omega)}^{\left(M_{p}\right) \prime}(\Gamma)$. Generalized analytic functions have the following quasi-analyticity property:

Theorem 7. Let $u \in \mathcal{O}_{v}^{\left(M_{p}\right)}\left(\widetilde{B}\left(e^{\omega}\right)\right)$. Suppose that for some $t<e^{\omega}$ and every $m \in \mathbb{N}$ there exist $C_{m}$ such that

$$
|u(x)| \leq C_{m} x^{m} \quad \text { for } 0<x \leq t .
$$

Then $u \equiv 0$.

Proof. By Theorem 6, $u(x)=\mathcal{T} S(x)$ for $x \in \widetilde{B}\left(e^{\omega}\right)$ with some $S \in L_{(\omega)}^{\left(M_{p}\right) \prime}(\Gamma)$. The assumption that $u$ is flat of arbitrary order $m \in \mathbb{N}$ on $(0, t)$ implies that $\mathcal{M}_{t} u \in \mathcal{O}(\mathbb{C})$. Since for every $R>0, L_{a, k}^{\left(M_{p}\right)}\left(\Gamma_{R}\right) \cap \mathcal{O}\left(\Gamma_{R}\right)=$ $L_{a}\left(\Gamma_{R}\right), \mathcal{M} u$ defines the zero element in $\widetilde{H}_{(\omega)}^{\left(M_{p}\right)}(\Gamma)$. Thus $S=0$ and $u \equiv 0$.

Theorem 8 (Strong quasi-analyticity principle). Let $-\pi / 2<\theta<\pi / 2$, $l_{\theta}=\left\{z=r e^{i \theta}: r>0\right\}$ and $F \in \mathcal{O}(\operatorname{Re} z>0)$. Suppose that for some $v \in \mathbb{R}$ and every $\kappa>0$ there exist $k<\infty$ and $C<\infty$ such that

$$
|F(z)| \leq C \exp \{v \operatorname{Re} z+M(k|z|)\} \quad \text { for } \operatorname{Re} z \geq \kappa .
$$

If for some $\tau>0$ and every $m \in \mathbb{N}$ there exists $C_{m}<\infty$ such that

$$
|F(z)| \leq C_{m} e^{-m \operatorname{Re} z} \quad \text { for } z \in l_{\theta}, \operatorname{Re} z \geq \tau,
$$

then $F \equiv 0$. 
Proof. Put $u(x)=F \circ \mu^{-1}(x)$ for $x \in \widetilde{B}(1)$. Then $u \in \mathcal{O}_{v}^{\left(M_{p}\right)}(\widetilde{B}(1))$. Set $t=e^{-\tau}$, let $\gamma_{t, \theta}$ be the set of $x \in \widetilde{B}(1)$ that satisfy

$$
x= \begin{cases}t \exp \{-i r \sin \theta\} & \text { for } 0 \leq r \leq \tau / \cos \theta, \\ \exp \{-r(\cos \theta+i \sin \theta)\} & \text { for } r \geq \tau / \cos \theta\end{cases}
$$

and observe that

$$
\mathcal{M}_{t} u(z)=\int_{\gamma_{t, \theta}} u(x) x^{-z-1} \quad \text { for } z \in \Omega_{v, \theta},
$$

where $\Omega_{v, \theta}=\{z \in \mathbb{C}: \operatorname{Re} z<v$ and $\sin \theta \operatorname{Im} z>\cos \theta(\operatorname{Re} z-v)\}$. Using (24) we infer that the right hand side of $(25)$ is defined for $z \in \mathbb{C}$. Thus, $\mathcal{M}_{t} u \in \mathcal{O}(\mathbb{C})$. As in the proof of Theorem 7 this implies that $u \equiv 0$ and hence $F \equiv 0$.

Remark 3. The conclusion of Theorem 8 does not hold if instead of (23) we assume that for every $\varepsilon>0$ and $\kappa>0$ there exists $C_{\varepsilon, \kappa}$ such that

$$
|F(z)| \leq C_{\varepsilon, \kappa} \exp \{v \operatorname{Re} z+\varepsilon|z|\} \quad \text { for } \operatorname{Re} z \geq \kappa .
$$

In this case the function $u=F \circ \mu^{-1}$ is the Taylor transform of an analytic functional with carrier at $\{\infty\}$ and need not be equal to zero.

Remark 4. The results of the paper can be easily extended to the $n$-dimensional case if $\Gamma$ is a cone of product type. The case of an arbitrary convex, proper cone in $\mathbb{R}^{n}$ is more difficult and will be studied in a subsequent paper.

\section{References}

[1] E. Hille, Analytic Function Theory, Vol. 2, Chelsea, New York, 1962.

[2] H. Komatsu, Ultradistributions, I. Structure theorems and a characterization, J. Fac. Sci. Univ. Tokyo 20 (1973), 25-105.

[3] -, Ultradistributions, II. The kernel theorem and ultradistributions with support in a submanifold, ibid. 24 (1977), 607-628.

[4] M. Langenbruch, Bases in spaces of ultradifferentiable functions with compact support, Math. Ann. 281 (1988), 31-42.

[5] G. Lysik, Generalized analytic functions and a strong quasi-analyticity principle, Dissertationes Math. 340 (1995), 195-200.

[6] S. Mandelbrojt, Séries adhérentes, régularisation de suites, applications, Gauthier-Villars, Paris, 1952.

[7] R. Meise and A. Taylor, Linear extension operators for ultradifferentiable functions of Beurling type on compact sets, Amer. J. Math. 111 (1989), 309-337.

[8] M. Morimoto, Analytic functionals with non-compact carrier, Tokyo J. Math. 1 (1978), 77-103.

[9] S. Pilipović, Tempered ultradistributions, Boll. Un. Mat. Ital. B (7) 2 (1988), 235-251. 
[10] C. Roumieu, Ultra-distributions définies sur $\mathbb{R}^{n}$ et sur certaines classes de variétés différentiables, J. Anal. Math. 10 (1962-63), 153-192.

[11] Z. Szmydt and B. Ziemian, The Mellin Transformation and Fuchsian Type Partial Differential Equations, Kluwer, Dordrecht, 1992.

[12] A. H. Zemanian, Generalized Integral Transformations, Interscience, 1969.

[13] B. Ziemian, Generalized analytic functions, Dissertationes Math., to appear.

INSTITUTE OF MATHEMATICS

POLISH ACADEMY OF SCIENCES

P.O. BOX 137

ŚNIADECKICH 8

00-950 WARSZAWA, POLAND

E-mail: LYSIK@IMPAN.GOV.PL

Reçu par la Rédaction le 7.5.1994

Révisé le 31.5.1995 